\title{
MAXIMAL FUNCTION ESTIMATES AND LOCAL WELL-POSEDNESS FOR THE GENERALIZED ZAKHAROV-KUZNETSOV EQUATION
}

\author{
FELIPE LINARES AND JOÃO P.G. RAMOS
}

\begin{abstract}
We prove a high-dimensional version of the Strichartz estimates for the unitary group associated to the free Zakharov-Kuznetsov equation. As a by-product, we deduce maximal estimates which allow us to prove local well-posedness for the generalized Zakharov-Kuznetsov equation in the whole subcritical case whenever $d \geq 4, k \geq 4$, complementing the recent results of Kinoshita [18, 19] and Herr-Kinoshita [15. Finally, we use some of those maximal estimates in order to prove pointwise convergence results for the flow of the generalized Zakharov-Kuznetsov equation in any dimension, in the same spirit of [5].
\end{abstract}

\section{INTRODUCTION}

In a manuscript published in 1974 [32], Zakharov and Kuznetsov deduced the equation

$$
\partial_{t} u+\partial_{x} \Delta u+u \partial_{x} u=0
$$

where $u=u(x, y, t)$ is a real-valued function, as means to describe propagation of ionicacoustic waves in a magnetized plasma. In [20, the authors derive (1.1) from the EulerPoisson system with magnetic field. Our main focus will remain, however, in the initial value problem (IVP) associated to the generalized version of this equations, as follows:

$$
\begin{cases}\partial_{t} u+\partial_{x} \Delta u+\partial_{x}\left(u^{k+1}\right)=0 & \text { on } \mathbb{R}^{d} \times \mathbb{R}, k \in \mathbb{Z}^{+}, \\ u(0)=u_{0} & \text { on } \mathbb{R}^{d},\end{cases}
$$

where the datum $u_{0}$ is taken to belong to an adequate Sobolev space $H^{s}\left(\mathbb{R}^{d}\right)$. This problem has attracted the attention of many authors since the work of Faminskii [10] on the $k=1$ case, who initially showed local well-posedness for $s \geq 1$ in the two-dimensional case. Eversince, many others have contributed in the two-dimensional case, among which we mention the work of Linares and Pastor [22] where it was proved local-wellposednes for $s>$ $3 / 4$ by employing smoothing effects. The works of Molinet and Pilod [25] and Grünrock and Herr 14, which proved local well-posedness by using the Fourier restriction method for $s>\frac{1}{2}$.

Still in the $k=1$ case, we also mention the work of Molinet-Pilod [25] and RibaudVento [27], which proved local and global well-posedness in $H^{s}\left(\mathbb{R}^{3}\right)$ for $s>1$. It was not until recently, however, in the works of Kinoshita [18] and Herr-Kinoshita [15] that well-posedness was obtained in the best possible range for the Picard iteration method: $s>-\frac{1}{4}$ if $d=2$ and $s>\frac{d-4}{2}$ for $d \geq 3$.

1991 Mathematics Subject Classification. Primary:42B25. Secondary: 35Q53, 42B37.

Key words and phrases. Maximal Functions, Zakharov-Kuznetsov equation. 
A key instrument in all references above in order to study this equation is estimating its free solutions. Indeed, let

$$
\begin{cases}\partial_{t} u+\partial_{x} \Delta u=0 & \text { on } \mathbb{R}^{d} \times \mathbb{R} ; \\ u(x, 0)=u_{0}(x) & \text { on } \mathbb{R}^{d}\end{cases}
$$

be the linear problem associated to the equation (1.1). We denote the group of operators associated to this problem by $U(t) u_{0}$ given by

$$
\widehat{U(t) u_{0}}(\xi, \eta)=e^{i t \xi\left(\xi^{2}+|\eta|^{2}\right)} \widehat{u_{0}}(\xi, \eta), \quad \xi \in \mathbb{R}, \eta \in \mathbb{R}^{d-1} .
$$

We remark that, as in the one-dimensional case of the KdV equation, this group possesses good maximal properties: indeed, if $d=2$, Linares and Pastor [22] proved that

$$
\left\|U(t) u_{0}\right\|_{L_{x}^{4} L_{y, T}^{\infty}} \lesssim\left\|u_{0}\right\|_{s}, s>3 / 4, T \in[0,1] .
$$

Later on, Grünrock [13] extended this estimate to arbitrary times. Also, Faminskii [10] showed that the same estimate holds with an $L_{x}^{2}$ norm:

$$
\left\|U(t) u_{0}\right\|_{L_{x}^{2} L_{y, t}^{\infty}} \lesssim\left\|u_{0}\right\|_{s}, s>3 / 4 .
$$

If $d=3$, Grünrock [12] proved that slightly stronger inequality holds in case $p=4$. Indeed, he proved that

$$
\left\|U(t) u_{0}\right\|_{L_{x, y}^{4} L_{t}^{\infty}} \lesssim\left\|u_{0}\right\|_{s}, s>3 / 4 .
$$

This implies, by Sobolev embedding, a maximal estimate of the form

$$
\left\|U(t) u_{0}\right\|_{L_{x}^{4} L_{y, t}^{\infty}} \lesssim\left\|u_{0}\right\|_{s},
$$

for $s>5 / 4$. Finally, complementing those results, we have the following time-weighted maximal estimate, proved by Ribaud-Vento [27]:

$$
\left\|t^{\alpha} U(t) u_{0}\right\|_{L_{x}^{2} L_{y, t}^{\infty}} \lesssim\left\|u_{0}\right\|_{s}, s>1,
$$

where we are allowed to take $\alpha \geq 3 / 8$.

In this note, we would like to address two main questions and assess their consequences, namely:

(i) (Space-time maximal estimate) Given $p \in(1, \infty)$, how large can $s \in \mathbb{R}$ be so that the estimate

$$
\left\|U(t) u_{0}\right\|_{L_{x}^{p} L_{y, t \in[0,1]}^{\infty}} \lesssim\left\|u_{0}\right\|_{s}
$$

holds for all $u_{0} \in \mathcal{S}\left(\mathbb{R}^{d}\right)$ ?

(ii) (Time-only maximal estimate) Given $p \in(1, \infty)$, how large can $s \in \mathbb{R}$ be so that the estimate

$$
\left\|U(t) u_{0}\right\|_{L_{x, y}^{p} L_{t \in[0,1]}^{\infty}} \lesssim\left\|u_{0}\right\|_{s}
$$

holds for all $u_{0} \in \mathcal{S}\left(\mathbb{R}^{d}\right)$ ?

Regarding those questions, we construct a simple set of counterexamples to give preliminary restrictions on such values of $s$. Indeed, the partial restrictions we have are the following: 
Proposition 1.1. Regarding the questions before, the following assertions hold:

(1) If (1.4) holds, then

$$
p \in[2,+\infty) \text { and } s \geq \frac{d}{2}-\frac{1}{p} .
$$

(2) If (1.5) holds, then

$$
p \in[2,+\infty) \text { and } s \geq \max \left\{d\left(\frac{1}{2}-\frac{1}{p}\right), \frac{3}{2 p}-\frac{d}{2}\left(\frac{1}{2}-\frac{1}{p}\right)\right\} .
$$

As a direct consequence, we are able to establish that some previous results in the literature are, in fact, sharp.

Theorem 1.2. Consider the group $\{U(t)\}_{t \in \mathbb{R}}$ the unitary group associated to (1.3).

(1) If $d=3$, then the estimates

$$
\left\|U(t) u_{0}\right\|_{L_{x}^{p} L_{y, t \in[0,1]}^{\infty}} \lesssim\left\|u_{0}\right\|_{s}
$$

hold whenever $s>\frac{3}{2}-\frac{1}{p}$ and $p \geq 2$. Moreover, this estimate is sharp, up to the endpoint $s=\frac{3}{2}-\frac{1}{p}$.

(2) If $d=2$, then the estimates

$$
\left\|U(t) u_{0}\right\|_{L_{x}^{p} L_{y, t \in[0,1]}^{\infty}} \lesssim\left\|u_{0}\right\|_{s}
$$

hold whenever either $s>1-\frac{1}{p}$ and $p \geq 4$ or $p \in[2,4)$ and $s>\frac{3}{4}$. Moreover, this estimate is sharp, up to the endpoint $s=1-\frac{1}{p}$, in case $p \in[4,+\infty)$, or up to the endpoint $s=\frac{3}{4}$ in case $p=2$.

In order to prove this theorem, we will use the already existing estimates for the group, which have been noted before (e.g. [10, 27, 12, 13] and others). The most interesting part of the proof of the result is the sharpness of the estimates when $d=2, p=2$, for which we actually resort to PDE methods.

Indeed, we show that, in case the estimate were true for some $s<\frac{3}{4}$ and $d=2, p=2$, the Picard iteration method would work for proving local well-posedness for the modified Zakharov-Kuznetsov equation in dimension 2 for some $s_{0}<\frac{1}{4}$. By a recent result of Kinoshita [19], however, we know that, similarly as in the KdV case, we can only prove local well-posedness by a contraction argument in case $s \geq \frac{1}{4}$. This contradiction establishes our result

These methods provide us, through Sobolev embedding and an argument by contradiction, with the $s>d\left(\frac{1}{2}-\frac{1}{p}\right)$ range for the second part of Proposition 1.1. In order to obtain the range mentioned in that statement, which does better in case $p \leq \frac{2(d+1)}{d}$, we employ results by Sjölin [30] and Rogers [28] on maximal estimates related to Schrödinger-like operators. See Section 2 below for more details.

Next, we establish Strichartz estimates for the unitary group $U(t)$ in higher dimensions. We remark that many of those estimates were obtained in the two and three dimensional cases in [22], 24]. In the current format, these follow closely [16], and are contained in the recent work of Schippa [29] for the critical line $\frac{2}{q}+\frac{2}{r}=1$. 
Proposition 1.3. Let $d \geq 3$, and $q, r \geq 2$. It holds that

$$
\left\|U(t) u_{0}\right\|_{L_{t \in[0,1]}^{r} L^{q\left(\mathbb{R}^{d}\right)}} \lesssim\left\|u_{0}\right\|_{H^{s}}
$$

where $\frac{2}{q}+\frac{2}{r} \leq 1$, and $s=d\left(\frac{1}{2}-\frac{1}{q}\right)-\frac{3}{r}$.

In particular, setting $q=r=4$ in this result implies a recent estimate by Herr-Kinoshita [15] (see also [19]), which enables the authors to prove subcritical local well-posedness for the Zakharov-Kuznetsov equation in any dimension:

$$
\left\|U(t) u_{0}\right\|_{L_{x, y,[0,1]}^{4}} \lesssim\left\|\langle\nabla\rangle^{\frac{d-3}{4}} u_{0}\right\|_{L^{2}}
$$

whenever $d \geq 3$. An application of Sobolev embedding in the $t$-variable readily implies the following maximal estimate:

$$
\left\|U(t) u_{0}\right\|_{L_{x, y}^{4} L_{[0,1]}^{\infty}} \lesssim\left\|u_{0}\right\|_{H^{s}},
$$

whenever $s>\frac{d}{4}$. Notice that this estimate is, in fact, sharp according to Proposition 1.1, as so is the other space-time maximal estimate which follows directly from the one above via Sobolev embedding, this time on the $y$-variable:

$$
\left\|U(t) u_{0}\right\|_{L_{x}^{4} L_{y,[0,1]}^{\infty}} \lesssim\left\|u_{0}\right\|_{H^{s}}
$$

for $s>\frac{d}{2}-\frac{1}{4}$.

Our final contribution is an usage of maximal estimates in order to prove well-posedness for the generalized Zakharov-Kuznetsov equation in higher dimensions:

$$
\begin{cases}\partial_{t} u+\partial_{x} \Delta u+\partial_{x}\left(u^{k+1}\right)=0 & \text { on } \mathbb{R}^{d} \times \mathbb{R} ; \\ u(x, 0)=u_{0}(x) & \text { on } \mathbb{R}^{d} .\end{cases}
$$

The modified and generalized versions of the Zakharov-Kuznetsov equation have been previously considered by many authors. In dimension 2, we mention briefly the works of Biagioni and Linares [2], Linares and Pastor [22, 21], and Farah, Linares and Pastor [11], which all contributed to the development of the topic. In particular, we mention the work of Ribaud and Vento [26] which proved local well-posedness for (1.7) whenever $k \geq 4$ in the full subcritical range $s>1-\frac{2}{k}$, and for $s>\frac{5}{12}$ for $k=3, s>\frac{1}{4}$ for $k=2$.

It was not until the works of Grünrock [12, 13] that local well-posedness was proved in the full subcritical range $s>\frac{1}{3}$ for the $k=3$ case in $d=2$, and also the full subcritical range $s>\frac{3}{2}-\frac{2}{k}$ was reached for any $k \in \mathbb{Z}, k \geq 2$ in $d=3$. Finally, we remark that the subcritical result $s>0$ for $k=2$ was recently shown by Kinoshita [18] not to hold if one demands smoothness of the flow map, which demonstrates, among other things, that the Ribaud-Vento result for $s>\frac{1}{4}$ in [27] was essentially sharp. Kinoshita also proved local well-posedness for small data in $s=\frac{1}{4}$ and full subcritical well-posedness $s>\frac{d}{2}-1$ in any dimension $d \geq 3$ for the modified Zakharov-Kuznetsov equation. He also shows small data global well-posedness for the critical space $H^{d / 2-1}$ for all $d \geq 3$.

Our result, as previously mentioned, complements the results of Herr-Kinoshita [15], Kinoshita [19] and Grünrock [12, 13]. 
Theorem 1.4. Let $d \geq 3$ and $k \geq 4$. Then there are function spaces $\mathcal{X}_{T}^{s}$ so that for each $u_{0} \in H^{s}\left(\mathbb{R}^{d}\right)$ with $s>\frac{d}{2}-\frac{2}{k}$, the IVP (1.7) has a unique solution

$$
u \in C\left([0, T]: H^{s}\left(\mathbb{R}^{d}\right) \cap \mathcal{X}_{T}^{s},\right.
$$

where $T=T\left(\left\|u_{0}\right\|_{s}\right)>0$. Moreover, the map $u_{0} \mapsto u$ from $H^{s}\left(\mathbb{R}^{d}\right)$ to $\mathcal{X}_{T}^{s} \cap C\left([0, T]: H^{s}\left(\mathbb{R}^{d}\right)\right)$ is locally Lipschitz continuous.

In order to prove this result, we will use (1.6) in conjunction with a local smoothing estimate. This is heavily inspired in the works of Ribaud and Vento [26, 27] and Grünrock [12, 13, and we only miss the full subcritical range in the $k=3$ case because of the lack of a sharp $L_{x}^{2} L_{y, T}^{\infty}$ estimate for $U(t)$; see the comments in the last section for more details.

Finally, we use the same $H^{\frac{d-3}{4}}+L_{x, y, T}^{4}$ estimate in order to prove a result about pointwise convergence of the flow to the initial data.

Theorem 1.5. Let $d, k \in \mathbb{N}$ as before. Let also $k \geq 2$ if $d \in\{2,3\}$ and $k \geq 4$ if $d \geq 4$, and $s>\max \left(\tilde{s}_{d}, \frac{d}{2}-\frac{2}{k}\right)$, where

$$
\tilde{s}_{d}= \begin{cases}\frac{1}{2} & \text { if } d=2,3 ; \\ \frac{d}{4} & \text { if } d \geq 4 .\end{cases}
$$

Then, for each initial datum $u_{0} \in H^{s}\left(\mathbb{R}^{d}\right)$, the unique solution $u \in C\left([0, T]: H^{s}\left(\mathbb{R}^{d}\right)\right)$ to the IVP (1.7) given by Theorem 1.4 converges pointwise to $u_{0}$; i.e.,

$$
\lim _{t \rightarrow 0} u(x, t)=u_{0}(x) \text {, for a.e. } x \in \mathbb{R}^{d} .
$$

Increasing attention has been given to such kinds of results in the recent literature, especially in the KdV case, where we mention the works of Erdoğan and Tzirakis both in the KdV and Schrödinger cases [8, 9], as well as the very recent results of Compaan, Lucà and Staffilani [5], which were responsible for establishing such results also in the context of the nonlinear Schrödinger equation in in higher dimensions in a sharp range, using the sharp pointwise convergence theorem for the Schrödinger flow [6, 7]. In our companion paper [23], we establish such a pointwise convergence result also in the case of the $L^{2}$-critical generalized Zakharov-Kuznetsov equation in three dimensions, i.e., $k=\frac{4}{3}$ in (1.7). There, our methods give us as range for well-posedness $s>\frac{3}{4}$, which coincides with the range in which the $H^{s} \rightarrow L_{x, y}^{4} L_{T}^{\infty}$ maximal estimates hold.

Notation and Organization. We use the modified Vinogradov equation $A \lesssim B$ several times to indicate that there is an absolute constant $C>0$ so that $A \leq C \cdot B$. We also use the original Vinogradov equation $A \ll B$ to denote that there is a (relatively) large constant $C$ with the property $A \leq C \cdot B$. We also use several times the notation $(-\Delta)^{s / 2} f=\langle\nabla\rangle^{s} f=\mathcal{F}^{-1}\left(\left(1+|\xi|^{2}+|\eta|^{2}\right)^{s / 2} \widehat{f}\right)$.

Finally, the paper is organized as follows. In Section 2, we discuss the counterxamples and the proof of Proposition 1.1 and Theorem [1.2, In Section 3, we prove Proposition 1.3. followed by a discussion on how the results in those sections imply well-posedness for the generalized Zakharov-Kuznetsov in higher dimensions and a proof of Theorem 1.5. 
Finally, in Section 4, we discuss some generalizations, remarks and open questions which arise naturally from our discussion.

\section{Proof of Theorem 1.2}

In this section, we will prove Theorem 1.2 by first showing, via an elementary counterexample, Proposition 1.1. We will then show that the necessary conditions provided by that proposition are, in fact, sharp in certain instances when $d=2,3$. The proof of such sharpness assumes previous results in the literature, and, as stated earlier, also an argument involving the modified Zakharov-Kuznetsov equation in two dimensions.

2.1. Proof of Proposition 1.1. As mentioned in the introduction, we construct a simple set of counterexamples to prove Proposition 1.1. First, define the Schwartz functions $\varphi_{j, k}$ on the Fourier side as

$$
\widehat{\varphi_{j, k}}(\xi, \eta)=\theta\left(2^{j} \xi\right) \psi\left(2^{-k} \eta\right),
$$

where $\theta$ is a smooth one-dimensional function supported at $[-4,-1 / 2] \cup[1 / 2,4]$, equal to 1 on $[-2,-1] \cup[1,2]$, and $\psi$ is a $(d-1)$-dimensional counterpart of $\theta$. If $j \geq-k$, a simple calculation implies that

$$
\left\|\varphi_{j, k}\right\|_{s}^{2} \sim 2^{(d-1) k-j} 2^{2 k s} .
$$

On the other hand, for $|y| \ll 2^{-k},|t| \ll \min \left(1,2^{j-2 k}\right)$, and $|x| \ll 2^{j}$, the Fourier transform definition of $U(t)$ implies that

$$
\left|U(t) \varphi_{j, k}(x, y)\right| \gtrsim 2^{(d-1) k-j} .
$$

This holds as the smallness assumptions are basically cancelling off the phase in the inverse Fourier transform definition of $U(t) \varphi_{j, k}$. Thus,

$$
2^{(d-1) k-j+\frac{j}{p}} \lesssim\left\|U(t) \varphi_{j, k}\right\|_{L_{x}^{p} L_{y, t}^{\infty}} \lesssim\left\|\varphi_{j, k}\right\|_{s}=2^{((d-1) k-j) / 2} 2^{k s} .
$$

In other words, $2^{\frac{d-1}{2} k+j(1 / p-1 / 2)} \lesssim 2^{k s}$. Here we have set ourselves the freedom to choose $j \geq-k$. If $p<2$, we can just let $j \rightarrow \infty$ to show that, in fact, no inequality of the type (1.4) can hold. For $p \geq 2$, the worst case scenario happens when $j=-k$, so that

$$
2^{\frac{d-1}{2} k+k(1 / 2-1 / p)} \lesssim 2^{k s}, \forall k \geq 1 \Longleftrightarrow s \geq \frac{d}{2}-\frac{1}{p}
$$

This proves the first part of 1.1. In order to prove the second one, assume that the inequality

$$
\left\|\sup _{t}\left|U(t) u_{0}\right|\right\|_{p} \lesssim\left\|u_{0}\right\|_{r}
$$

holds with $p>1$. By Sobolev embedding, this estimate implies that (1.4) holds for all $s>r+\frac{d-1}{p}$. Therefore,

$$
r \geq d\left(\frac{1}{2}-\frac{1}{p}\right)
$$

This finishes the proof of the first lower bound on the second part. In order to prove the $s>\frac{3}{2 p}-\frac{n}{2}\left(\frac{1}{2}-\frac{1}{p}\right)$ restriction, we shall use two different results. The first of them is the following bound obtained by Sjölin in [30, Theorem 1]. 
Theorem A. Suppose that $\Omega: \mathbb{R}^{d} \rightarrow \mathbb{R}$ is a smooth homogeneous polynomial of degree $\geq 1$. Then the estimate

$$
\left\|\sup _{t \in[0,1]}\left|e^{i t \Omega(D)} f\right|\right\|_{L^{p}\left(B^{d}(0,1)\right)} \lesssim\|f\|_{H^{s}\left(\mathbb{R}^{d}\right)}
$$

can only hold if $s+\frac{n-1}{2 p} \geq \frac{n}{4}$. Here, we let $\widehat{\Omega(D) f}(\zeta)=\Omega(\zeta) \widehat{f}(\zeta)$.

As an immediate corollary, we see that the bound

$$
\left\|\sup _{t \in[0,1]}|U(t) f|\right\|_{L^{p}\left(B^{d}(0,1)\right)} \lesssim\|f\|_{H^{s}\left(\mathbb{R}^{d}\right)}
$$

can only hold if $s+\frac{n-1}{2 p} \geq \frac{n}{4}$. In order to pass from the unit ball to the whole euclidean space, we must use the following result by Rogers [28, Theorem 1.3]:

Theorem B. Let $p \geq 2$ and $\Omega: \mathbb{R}^{d} \rightarrow \mathbb{R}$ be a smooth function so that, for some integer $m \geq 2$, it holds that $\left|D^{\alpha} \Omega(\zeta)\right| \lesssim_{\alpha}|\zeta|^{m-|\alpha|}$ for all multiindices $|\alpha| \leq 2$, and that $|\nabla \Omega(\zeta)| \gtrsim$ $|\zeta|^{m-1}$. Then the local maximal estimate

$$
\left\|\sup _{t \in[0,1]}\left|e^{i t \Omega(D)} f\right|\right\|_{L^{p}\left(B^{d}(0,1)\right)} \lesssim\|f\|_{H^{s}\left(\mathbb{R}^{d}\right)}
$$

holds for all $s>s_{0}$ if and only if the global maximal estimate

$$
\left\|\sup _{t \in[0,1]}\left|e^{i t \Omega(D)} f\right|\right\|_{L^{p}\left(\mathbb{R}^{d}\right)} \lesssim\|f\|_{H^{s}\left(\mathbb{R}^{d}\right)}
$$

holds for all $s>m s_{0}-(m-1) d\left(\frac{1}{2}-\frac{1}{p}\right)$.

In our case, it is easy to see that $\Omega(\xi, \eta)=\xi\left(\xi^{2}+|\eta|^{2}\right)$ satisfies the hypotheses above with $m=3$. Therefore, Theorems $\mathrm{A}$ and $\mathrm{B}$ imply together that the time-only maximal estimate 1.5 can only hold whenever $s>3\left(\frac{d}{4}-\frac{d-1}{2 p}\right)-2 d\left(\frac{1}{2}-\frac{1}{p}\right)=\frac{3}{2 p}-\frac{d}{2}\left(\frac{1}{2}-\frac{1}{p}\right)$. This completes our proof.

2.2. Analysis of the three-dimensional maximal estimate. In three-dimensions, the existing results are (essentially) sharp and completely compatible with the counterexamples given above. Indeed, it is not complicated to prove that, for $p \rightarrow \infty$, Faminskii's methods imply that

$$
\left\|U(t) u_{0}\right\|_{L_{x, y,[0,1]}^{\infty-}} \lesssim\left\|u_{0}\right\|_{s}, s>3 / 2
$$

Indeed, (2.1) follows by a simple Sobolev embedding argument:

$$
\left\|U(t) u_{0}\right\|_{L_{x, y, t \in[0,1]}^{N}} \lesssim\left\|\left\langle\nabla_{x, y}\right\rangle^{\frac{3(N-2)}{2 N}} U(t) u_{0}\right\|_{L_{[0,1]}^{N} L_{x, y}^{2}} \leq\left\|u_{0}\right\|_{H^{\frac{3(N-2)}{2 N}}} .
$$

We are then allowed to directly interpolate this bound with Ribaud-Vento [27, Proposition 3.3], which states

$$
\left\|U(t) u_{0}\right\|_{L_{x}^{2} L_{y,[0,1]}^{\infty}} \lesssim\left\|u_{0}\right\|_{H^{s}}, s>1 .
$$

This yields the result directly by letting $N \rightarrow \infty$. 
Alternatively, one can also follow Faminskii's approach. We are then required to estimate functions of the form

$$
I_{k}(x, y, t)=\int_{\mathbb{R}^{3}} e^{i\left(x \xi+y \cdot \eta+t \xi\left(\xi^{2}+|\eta|^{2}\right)\right)} \phi\left(2^{-k}(\xi, \eta)\right) \mathrm{d} \xi \mathrm{d} \eta,
$$

where $\phi$ is a smooth indicator function of the annulus $\{|(\xi, \eta)| \sim 1\}$. We clearly have $\left\|I_{k}\right\|_{L_{x, y, t}^{\infty}} \leq 2^{3 k}$. By [27, Lemma 3.3], we have $\left\|I_{k}\right\|_{L_{x}^{1} L_{y, t}^{\infty}} \lesssim \operatorname{poly}(k) 2^{2 k}$. Interpolation yields

$$
\left\|I_{k}\right\|_{L_{x}^{p} L_{y, t}^{\infty}} \lesssim \operatorname{poly}(k) 2^{2 k+(1-\theta) k}
$$

where $p=\frac{1}{\theta}$. An application of the $T T^{*}$ method and a Littlewood-Paley analysis yields

$$
\left\|U(t) u_{0}\right\|_{L_{x}^{q} L_{y, t}^{\infty}} \lesssim\left\|u_{0}\right\|_{s}, s>1+\frac{1-\theta}{2}, \text { for } q=\frac{2}{\theta} .
$$

On the other hand, inequality (1.4) only holds if $s \geq \frac{3}{2}-\frac{1}{p}$. Setting $p=q=\frac{2}{\theta}$ gives us that the result is, indeed, sharp, up to the endpoint, as we wished.

2.3. Analysis of the two-dimensional maximal estimate. For the case of $d=2$, we have a distinction to make:

If $p \geq 4$, then the counterexample we gave yields the sharp bound, as Linares-Pastor and Grünrock [22, 12] show that

$$
\left\|U(t) u_{0}\right\|_{L_{x}^{4} L_{y, t}^{\infty}} \lesssim\left\|u_{0}\right\|_{s}, s>3 / 4 .
$$

By the Sobolev embedding argument from the previous section, one easily sees that the range of $s$ one gets matches with that of our counterexample if $p \geq 4$, except for the endpoint $s=1-\frac{1}{p}$.

On the other hand, if $p \in[2,4)$, the subject is much more delicate. Indeed, we will show that Faminskii's result

$$
\left\|U(t) u_{0}\right\|_{L_{x}^{2} L_{y, t}^{\infty}} \lesssim\left\|u_{0}\right\|_{s}
$$

for $s>3 / 4$, is optimal, up to the endpoint $s=3 / 4$. Instead of building an explicit counterexample, we argue by contradiction.

In fact, suppose that (2.2) holds for $s>s_{0}, 3 / 4>s_{0}$. We follow Ribaud-Vento's ideas for the well-posedness of the modified Zakharov-Kuznetsov equation [26]. More precisely, we wish to construct a solution to

$$
\begin{cases}\partial_{t} u+\partial_{x} \Delta u+\partial_{x}\left(u^{3}\right)=0 & \text { on } \mathbb{R} \times \mathbb{R}^{2}, \\ u(x, 0)=u_{0}(x) & \text { on } \mathbb{R}^{2}\end{cases}
$$

for all $u_{0} \in H^{s}$, with $s$ to be specified in a while. The idea is that, if $s$ is sufficiently small and we are able to prove that the data-to-solution map $u_{0} \mapsto u$ above is, in fact, smooth, then we will directly contradict Kinoshita's result, which shows that the data-to-solution mapping cannot possess $C^{3}$ regularity in the case of the modified Zakharov-Kuznetsov equation in dimension 2.

To that extent, define the auxiliary norms

$$
\|u\|_{Y_{T}^{s}}=\|u\|_{L_{T}^{\infty} H_{x, y}^{s}}+\left\|\left\langle\nabla_{x, y}\right\rangle^{s+1} u\right\|_{L_{x}^{\infty} L_{y, T}^{2}}+\left\|\left\langle\nabla_{x, y}\right\rangle^{s-s_{0}^{+}} u\right\|_{L_{x}^{2} L_{y, T}^{\infty}},
$$


and, after that, the space in which we wish to construct a solution as $X_{T}^{s}=\{u \in$ $\left.C\left([0, T] ; H^{s}\left(\mathbb{R}^{d}\right)\right),\|u\|_{X_{T}^{s}}<\infty\right\}$, where

$$
\|u\|_{X_{T}^{s}}=\|\| \Delta_{k} u\left\|_{Y_{T}^{s}}\right\|_{\ell^{2}(\mathbb{N})} .
$$

Here $\mathcal{F}_{x, y}\left(\Delta_{k} u\right)(\xi, \eta, t)=\phi\left(2^{-k}(\xi, \eta)\right) \mathcal{F}_{x, y} u(\xi, \eta, t)$ denotes the $k$-th Littlewood-Paley projection in frequency. Because $U(t)$ is unitary, the presence of Kato smoothing and the fact that we supposed that (2.2) holds for $s>s_{0}$, then

$$
\left\|U(t) u_{0}\right\|_{X_{T}^{s}} \lesssim\left\|u_{0}\right\|_{s}, \forall s \in \mathbb{R} .
$$

Following the Duhamel formulation of (2.3), we wish to prove that the map

$$
F(u)=U(t) u_{0}+\int_{0}^{t} U\left(t-t^{\prime}\right) \partial_{x}\left(u^{3}\right) \mathrm{d} t^{\prime}
$$

has a fixed point in the space $E(T, a)$ of functions whose $X_{T}^{s}$-norm is at most $a$. The linear part is controlled because of (2.4), so we focus on the non-linear part. By the methods from both [26] and [27, the non-linear part of (2.5) can be estimated by

$$
\left\|\int_{0}^{t} U\left(t-t^{\prime}\right) \partial_{x}\left(u^{3}\right) \mathrm{d} t^{\prime}\right\|_{X_{T}^{s}} \lesssim\left\|2^{s k}\right\| \Delta_{k}\left(u^{3}\right)\left\|_{L_{x}^{1} L_{y, T}^{2}}\right\|_{\ell^{2}(\mathbb{N})} .
$$

Our goal is to estimate the right-hand side in terms of a power of the $X_{T}^{s}$ norm of $u$. To that extent, we notice that the definition of $Y_{T}^{s}$ gives us

$$
2^{(s+1) k}\left\|\Delta_{k} u\right\|_{L_{x}^{\infty} L_{y, T}^{2}} \lesssim\left\|\Delta_{k} u\right\|_{Y_{T}^{s}}
$$

and

$$
2^{\left(s-s_{0}^{+}\right) k}\left\|\Delta_{k} u\right\|_{L_{x}^{2} L_{y, T}^{\infty}} \lesssim\left\|\Delta_{k} u\right\|_{Y_{T}^{s}}
$$

Interpolation yields then

$$
2^{\alpha k}\left\|\Delta_{k} u\right\|_{L_{x}^{p} L_{y, T}^{q}} \lesssim\left\|\Delta_{k} u\right\|_{Y_{T}^{s}}
$$

whenever $\frac{1}{p}=\frac{1-\theta}{2}, \frac{1}{q}=\frac{\theta}{2}$, and $\alpha=\left(s+\left(1+s_{0}\right) \theta-s_{0}\right)^{-}$. In particular, taking $\theta={\frac{s_{0}}{1+s_{0}}}^{+}$, we get

$$
2^{s k}\left\|\Delta_{k} u\right\|_{L_{x}^{p_{0}^{+}} L_{y, T}^{q_{0}^{-}}} \lesssim\left\|\Delta_{k} u\right\|_{Y_{T}^{s}}
$$

with $q_{0}=\frac{2\left(1+s_{0}\right)}{s_{0}}, p_{0}=2\left(1+s_{0}\right)$. Employing the paraproduct decomposition

$$
\Delta_{k}\left(u^{3}\right)=\Delta_{k}\left[\left(P_{0} u\right)^{3}+\sum_{j \geq k}\left(\Delta_{j+1} u\right)\left(\left(P_{j+1} u\right)^{2}+\left(P_{j} u\right)\left(P_{j+1} u\right)+\left(P_{j} u\right)^{2}\right)\right]
$$

together with Hölder's inequality several times shows that

$$
\left\|\Delta_{k}\left(u^{3}\right)\right\|_{L_{x}^{1} L_{y, T}^{2}} \lesssim \sum_{l \geq k}\left\|\Delta_{l} u\right\|_{L_{x}^{p_{0}^{+}} L_{y, T}^{q_{0}^{-}}}\left\|P_{l} u\right\|_{L_{x}^{2 p_{1}^{-}} L_{y, T}^{2 q_{1}^{+}}}^{2}+\left\|P_{0} u\right\|_{L_{x}^{p_{0}^{+}} L_{y, T}^{q_{0}^{-}}}\left\|P_{0} u\right\|_{L_{x}^{2 p_{1}^{-}} L_{y, T}^{2 q_{1}^{+}}}^{2}
$$

where $p_{1}=\frac{p_{0}}{p_{0}-1}, q_{1}=\frac{2 q_{0}}{q_{0}-2}$. Here we denote by $P_{j} u$ the smooth (space) frequency projection of $u$ onto the ball of center zero and radius $\sim 2^{j}$ given by $P_{j}=\sum_{l \leq j}\left(\Delta_{l} u\right)$. We then 
take $\theta={\frac{1}{q_{1}}}^{-}$on (2.6) . Notice that $q_{1}=\frac{4\left(1+s_{0}\right) / s_{0}}{2 / s_{0}}=2\left(1+s_{0}\right)=p_{0}$. This implies that

$$
2^{\left(s+\frac{1}{2}-s_{0}\right)^{-} k}\left\|\Delta_{k} u\right\|_{L_{x}^{2 p_{1}^{-}}} L_{y, T}^{2 q_{1}^{+}} \lesssim\left\|\Delta_{k} u\right\|_{Y_{T}^{s}} .
$$

We can therefore estimate

$$
\left\|P_{j} u\right\|_{L_{x}^{2 p_{1}^{-}} L_{y, T}^{2 q_{1}^{+}}} \lesssim \sum_{k \leq j}\left\|\Delta_{k} u\right\|_{L_{x}^{2 p_{1}^{-}} L_{y, T}^{2 q_{1}^{+}}} \lesssim\left(\sum_{k \leq j} 2^{-\left(s+\frac{1}{2}-s_{0}\right)^{-} k}\right)\|u\|_{X_{T}^{s}} .
$$

The sum above converges as long as $s>s_{0}-\frac{1}{2}$. Therefore,

$$
\left\|P_{j} u\right\|_{L_{x}^{2 p_{1}^{-}} L_{y, T}^{2 q_{1}^{+}}} \lesssim\|u\|_{X_{T}^{s}} .
$$

In order to finish the iteration argument, we note the simple estimate

$$
2^{(s-1)^{+} j}\left\|\Delta_{j} u\right\|_{L_{x, y, T}^{N}} \lesssim T^{\delta_{N}}\|u\|_{X_{T}^{s}}
$$

which holds by Sobolev embedding and Hölder's inequality, for $N \gg 1$. Using the bound on the right hand side of (2.7) and interpolating with (2.8) for $N$ arbitrarily large, we get that

$$
\left\|2^{s k}\right\| \Delta_{k}\left(u^{3}\right)\left\|_{L_{x}^{1} L_{y, T}^{2}}\right\|_{\ell^{2}(\mathbb{N})} \lesssim T^{\delta}\|u\|_{X_{T}^{s}}^{2}\left\|\left(1_{j \geq 0} 2^{-s j}\right) *\right\|\left(\Delta_{j} u\right)\left\|_{Y_{T}^{s}}\right\|_{\ell^{2}(\mathbb{N})} .
$$

By the discrete version of Young's convolution inequality, the last expression is bounded by $T^{\delta}\|u\|_{X_{T}^{s}}^{3}$, with $\delta>0$. Notice that we can run this argument whenever $s>s_{0}-1 / 2$. Therefore, by Picard's iteration method, we would obtain the local well-posedness of (2.3) in $H^{s}, s>s_{0}-1 / 2$. Moreover, we actually show that, if $s>s_{0}-1 / 2$, there is $\delta>0$ so that

$$
\|F(u)\|_{X_{T}^{s}} \leq c\left\|u_{0}\right\|_{s}+C T^{\delta}\|u\|_{X_{T}^{s}}^{3} .
$$

As the nonlinearity in (2.3) is smooth, a standard technique using the Duhamel formulation shows that the data-to-solution map given by the solution of the IVP (2.3) is, in fact, $C^{\infty}$-smooth for all $s>s_{0}-1 / 2$. But the recent result by Kinoshita [19, Theorem 1.3] proved that the data-to-solution map induced by (2.3) is not $C^{3}$-smooth for $s<1 / 4$, and thus, as $s_{0}<3 / 4$, this is a contradiction, which finally implies that $s_{0} \geq \frac{3}{4}$, as originally wished.

\section{Proof of Theorem 1.4}

Finally, we prove the local well-posedness result for the generalized Zakharov-Kuznetsov equation in higher dimensions.

3.1. Proof of Proposition 1.3. In this subsection, we prove some auxiliary Strichartz estimates, which will enable us to prove the local well-posedness results for the generalized Zakharov-Kuznetsov equation in any dimension $d>3$, given that $k \geq 3$.

We start by proving a dispersion estimate for the group $U(t)=: e^{-t \partial_{x} \Delta}$.

Proposition 3.1. Let $d \geq 3$ and $p \in[2,+\infty)$. Then for each $t \in \mathbb{R}$ it holds that

$$
\left\|e^{-t \partial_{x} \Delta} f\right\|_{L^{p}} \lesssim|t|^{-2\left(\frac{1}{2}-\frac{1}{p}\right)}\left\|\left\langle\nabla_{x, y}\right\rangle^{(d-3)\left(1-\frac{2}{p}\right)} f\right\|_{L^{p^{\prime}}},
$$



for each $f \in \mathcal{S}\left(\mathbb{R}^{d}\right)$.

Proof. We first bound the left-hand side of the conclusion of Proposition 3.1 as

$$
\|U(t) f\|_{L^{p}} \lesssim\left(\sum_{k \geq 0}\left\|U(t) \Delta_{k} f\right\|_{L^{p}}^{2}\right)^{1 / 2},
$$

so that it suffices to bound each summand on the right-hand side of (3.1). In order to do so, it suffices to prove that

$$
\left\|U(t) \Delta_{0} f\right\|_{L^{p}} \lesssim|t|^{-2\left(\frac{1}{2}-\frac{1}{p}\right)}\|f\|_{L^{p^{\prime}}} .
$$

Indeed, suppose (3.2) holds, and let $f(x)=\frac{1}{2^{k d}}\left(\tilde{\Delta}_{k} g\right)\left(x / 2^{k}\right)$ above. A simple computation then shows that

$$
U(t) \Delta_{0} f(x)=2^{-k d} U\left(t / 2^{3 k}\right) \Delta_{k} g\left(x / 2^{k}\right)
$$

Therefore,

$$
\begin{aligned}
& 2^{k d\left(\frac{1}{p}-1\right)}\left\|U\left(t / 2^{3 k}\right) \Delta_{k} g\right\|_{L^{p}}=\left\|U(t) \Delta_{0} f\right\|_{L^{p}} \\
\lesssim & |t|^{-2\left(\frac{1}{2}-\frac{1}{p}\right)}\|f\|_{L^{p^{\prime}}}=|t|^{-2\left(\frac{1}{2}-\frac{1}{p}\right)} 2^{k d\left(\frac{1}{p^{\prime}}-1\right)}\left\|\tilde{\Delta}_{k} g\right\|_{L^{p^{\prime}}}
\end{aligned}
$$

for all $t \in \mathbb{R}$. Rearranging terms, we get that

$$
\begin{aligned}
\left\|U\left(t^{\prime}\right) \Delta_{k} g\right\|_{L^{p}} & \lesssim\left|t^{\prime}\right|^{-2\left(\frac{1}{2}-\frac{1}{p}\right)} 2^{k(d-3)\left(1-\frac{2}{p}\right)}\left\|\tilde{\Delta}_{k} g\right\|_{L^{p^{\prime}}} \\
& \sim\left|t^{\prime}\right|^{-2\left(\frac{1}{2}-\frac{1}{p}\right)}\left\|\left\langle\nabla_{x, y}\right\rangle^{k(d-3)\left(1-\frac{2}{p}\right)} \tilde{\Delta}_{k} g\right\|_{L^{p^{\prime}}},
\end{aligned}
$$

for all $t^{\prime} \in \mathbb{R}$. By (3.1), we have then

$$
\begin{aligned}
\|U(t) g\|_{L^{p}} & \lesssim|t|^{-2\left(\frac{1}{2}-\frac{1}{p}\right)}\left(\sum_{k \geq 0}\left\|\left\langle\nabla_{x, y}\right\rangle^{k(d-3)\left(1-\frac{2}{p}\right)} \tilde{\Delta}_{k} g\right\|_{L^{p^{\prime}}}\right) \\
& \lesssim|t|^{-2\left(\frac{1}{2}-\frac{1}{p}\right)}\left\|\left(\sum_{k \geq 0}\left|\Delta_{k}\left\langle\nabla_{x, y}\right\rangle^{k(d-3)\left(1-\frac{2}{p}\right)} g\right|^{2}\right)^{1 / 2}\right\|_{L^{p^{\prime}}} \\
& \lesssim|t|^{-2\left(\frac{1}{2}-\frac{1}{p}\right)}\left\|\left\langle\nabla_{x, y}\right\rangle^{k(d-3)\left(1-\frac{2}{p}\right)} g\right\|_{L^{p^{\prime}}},
\end{aligned}
$$

by the Littlewood-Paley theorem.

In order to prove (3.2), we prove the endpoints $p=2$ and $p=\infty$ and interpolate. For the $p=2$ case, we simply use Plancherel's theorem to obtain

$$
\left\|U(t) \Delta_{0} f\right\|_{L^{2}} \lesssim\|f\|_{L^{2}}
$$

For the $p=\infty$ case, we rewrite

$$
\begin{aligned}
U(t) \Delta_{0} f(x) & =\mathcal{F}^{-1}\left(\psi(|\xi|) e^{i t \xi_{1}|\xi|^{2}} \widehat{f}(\xi)\right)(x)=\int_{\mathbb{R}^{d}} \psi(|\xi|) e^{i t \xi_{1}|\xi|^{2}} \widehat{f}(\xi) e^{-i x \cdot \xi} \mathrm{d} \xi \\
& =\int_{\mathbb{R}^{d}} f(y)\left(\int_{\mathbb{R}^{d}} \psi(|\xi|) e^{i t \xi_{1}|\xi|^{2}+i(x-y) \cdot \xi} \mathrm{d} \xi\right) \mathrm{d} y
\end{aligned}
$$


by Fourier inversion. Taking the modulus inside and using the following lemma yields the proposition:

Lemma 3.2 (Proposition 2.1 in [29]). Let $\psi: \mathbb{R}^{d} \rightarrow \mathbb{R}$ be a smooth radial function supported in $B_{d}(0,2) \backslash B_{d}(0,1 / 2)$. Then it holds that

$$
\left|\int_{\mathbb{R}^{d}} \psi(|\xi|) e^{i t \xi_{1}|\xi|^{2}+i z \cdot \xi} \mathrm{d} \xi\right| \leq C|t|^{-1}
$$

where $C>0$ does not depend on $z \in \mathbb{R}^{d}$.

See [29] (and alternatively [16, Proposition 3.1]) for a proof.

As previously remarked, we are now done. Indeed, it then holds that

$$
\left|U(t) \Delta_{0} f(x)\right| \lesssim|t|^{-1}\|f\|_{L^{1}},
$$

which, by the previous considerations, finishes our proof.

Proof of Proposition 1.3. Now that we have Proposition 3.1, we can perform the usual $T T^{*}$ method. First, consider the $\frac{2}{q}+\frac{2}{r}=1$ line. By the usual duality arguments, the conclusion follows if we prove that

$$
\left\|\int(-\Delta)^{s} U\left(t-t^{\prime}\right) F\left(\cdot, t^{\prime}\right) \mathrm{d} t^{\prime}\right\|_{L_{t \in[0,1]}^{r} L^{q}\left(\mathbb{R}^{d}\right)} \lesssim\|F\|_{L_{t \in[0,1]}^{r^{\prime}} L^{q^{\prime}}\left(\mathbb{R}^{d}\right)},
$$

for all $F \in \mathcal{S}\left(\mathbb{R}^{d+1}\right)$. As we have that $\frac{1}{r}=\frac{1}{2}-\frac{1}{q}$, the value of $s=(d-3)\left(\frac{1}{2}-\frac{1}{q}\right)$ allows us to use the Hardy-Sobolev inequality:

$$
\begin{aligned}
\left\|\int(-\Delta)^{s} U\left(t-t^{\prime}\right) F\left(\cdot, t^{\prime}\right) \mathrm{d} t^{\prime}\right\|_{L_{t \in[0,1]}^{r} L^{q}\left(\mathbb{R}^{d}\right)} & \lesssim\left\|\int\right\|(-\Delta)^{s} U\left(t-t^{\prime}\right) F\left(\cdot, t^{\prime}\right)\left\|_{L^{q}\left(\mathbb{R}^{d}\right)} \mathrm{d} t^{\prime}\right\|_{L^{r}} \\
& \lesssim \| \int \frac{\left\|F\left(\cdot, t^{\prime}\right)\right\|_{L^{q^{\prime}\left(\mathbb{R}^{d}\right)}} \mathrm{d} t^{\prime} \|_{L^{r}}}{\left|t-t^{\prime}\right|^{1-\frac{2}{q}}} \\
& \lesssim\|F\|_{L_{[0,1]}^{r^{\prime}} L^{q^{\prime}}\left(\mathbb{R}^{d}\right)^{.}}
\end{aligned}
$$

For the $\frac{2}{q}+\frac{2}{r}<1$ case, let $\tilde{q}$ be such that $\frac{2}{\tilde{q}}+\frac{2}{r}=1$, employ the strategy above for such $\tilde{q}$ and then apply the sharp version of the Sobolev embedding theorem. This finishes the proof.

3.2. Linear Estimates. We will need, besides the Strichartz estimates from the previous subsection, some linear estimates to free solutions to (1.3). We remark that most of them are just direct adaptations of the low-dimensional settings, and thus the brevity in their proofs. See the mentioned references throughout the text for more details.

Proposition 3.3 (Kato smoothing). Let $u_{0} \in \mathcal{S}\left(\mathbb{R}^{d}\right)$ and $U(t)$ be as before. Then it holds that

$$
\left\|\nabla U(t) u_{0}\right\|_{L_{x}^{\infty} L_{y, t}^{2}} \lesssim\left\|u_{0}\right\|_{L^{2}} .
$$

Proof. This proof is basically a remake of the result for the KdV [17] and of the two- and three-dimensional cases [10, 27]. 
In fact, we perform the change of variables $\vartheta=\xi\left(\xi^{2}+|\eta|^{2}\right)=h_{\eta}(\xi)$ in the Fourier definition of $U(t) u_{0}$. This yields

$$
U(t) u_{0}=\mathcal{F}_{\vartheta, \eta}^{-1}\left(e^{i x\left(h_{\eta}\right)^{-1}(\vartheta)}\left(h_{\eta}^{-1}\right)^{\prime}(\vartheta) \widehat{u_{0}}\left(h_{\eta}(\vartheta), \eta\right)\right)(y, t) .
$$

Using now Plancherel and inverting the change of variables, we obtain

$$
\left\|U(t) u_{0}\right\|_{L_{y, t}^{2}}=\left\|\left(h_{\eta}^{-1}\right)^{\prime}(\vartheta) \widehat{u_{0}}\left(h_{\eta}(\vartheta), \eta\right)\right\|_{L_{\vartheta, \eta}^{2}}=\left\|\left|h_{\eta}^{\prime}(\xi)\right|^{-1 / 2} \widehat{u_{0}}(\xi, \eta, \tau)\right\|_{L_{\xi, \eta}^{2}} .
$$

By noticing that $h_{\eta}^{\prime}(\xi)=3 \xi^{2}+|\eta|^{2} \sim \xi^{2}+\eta^{2} \sim \mathcal{F}(-\Delta)$, by applying the expression above to $u_{0}=\nabla v_{0}$ we get to

$$
\left\|\nabla U(t) v_{0}\right\|_{L_{y, t}^{2}} \lesssim\left\|v_{0}\right\|_{L_{x, y}^{2}},
$$

and thus taking the supremum of the left-hand side in $x$ gives us the result.

Proposition 3.4 (Maximal estimate). Let $d \geq 3$. It holds that

$$
\left\|U(t) u_{0}\right\|_{L_{x}^{4} L_{y, t}^{\infty}} \lesssim\left\|u_{0}\right\|_{H^{s}}
$$

where $s>\frac{d}{2}-\frac{1}{4}$.

Proof. As previously mentioned, Proposition 1.3 implies directly that

$$
\left\|U(t) u_{0}\right\|_{L_{x, y, t}^{4}} \lesssim\left\|\langle\nabla\rangle^{\frac{d-3}{4}} u_{0}\right\|_{L^{2}} .
$$

Now, if we use Sobolev embedding on the $t$-variable and the fact that $\left(\partial_{t}\right)^{r} U(t)=$ $\left(\partial_{x} \Delta\right)^{r} U(t)$ (which follows from the fact that the time-space Fourier support of $U(t) u_{0}$ is the surface $\left.\left\{\tau=\xi\left(\xi^{2}+|\eta|^{2}\right)\right\}\right)$, followed by Sobolev embedding in the $y$-variable, we obtain

$$
\left\|U(t) u_{0}\right\|_{L_{x}^{4} L_{y, t}^{\infty}} \lesssim\left\|\left(\partial_{x} \Delta\right)^{1 / 4^{+}}\langle\nabla\rangle^{\frac{d-2}{2}} u_{0}\right\|_{L^{2}} .
$$

A simple computation on the Fourier side shows that the last expression is bounded by $\left\|\langle\nabla\rangle^{s} u_{0}\right\|_{L^{2}}$ whenever $s>\frac{d}{2}-\frac{1}{4}$, as desired.

With these estimates in hands, we prove some retarded estimates which will be key to perform the fix-point argument.

Proposition 3.5. Let $f \in \mathcal{S}\left(\mathbb{R}^{d+1}\right)$. Then it holds that

$$
\left\|\nabla \int_{0}^{t} U\left(t-t^{\prime}\right) f\left(\cdot, t^{\prime}\right) \mathrm{d} t^{\prime}\right\|_{L_{T}^{\infty} L_{x, y}^{2}} \lesssim\|f\|_{L_{x}^{1} L_{y, T}^{2}} .
$$

Proof. We compose the dual version of Proposition 3.3 with the fact that $U(t)$ is an unitary group; this implies that

$$
\left\|\nabla \int_{0}^{T} U\left(t-t^{\prime}\right) f\left(\cdot, t^{\prime}\right) \mathrm{d} t^{\prime}\right\|_{L_{x, y}^{2}} \lesssim\|f\|_{L_{x}^{2} L_{y, T}^{2}} .
$$

The proposition then follows if applied to $\tilde{f}\left(z, t^{\prime}\right)=1_{[0, t]}\left(t^{\prime}\right) f\left(z, t^{\prime}\right)$ and taking the supremum on $t \in[0, T]$ of the left-hand side.

Proposition 3.6. Let $f \in \mathcal{S}\left(\mathbb{R}^{d+1}\right)$. Then it holds that

$$
\left\|\nabla^{2} \int_{0}^{t} U\left(t-t^{\prime}\right) f\left(\cdot, t^{\prime}\right) \mathrm{d} t^{\prime}\right\|_{L_{x}^{\infty} L_{y, T}^{2}} \lesssim\|f\|_{L_{x}^{1} L_{y, T}^{2}} .
$$


Proof. The proof of this proposition follows, essentially, the same lines of [27, Proposition 3.6].

In fact, we start out by writing $\nabla^{2} \int_{0}^{t} U\left(t-t^{\prime}\right) f\left(\cdot, t^{\prime}\right) \mathrm{d} t^{\prime}$ as the sum

$$
\frac{1}{2} \nabla^{2} \int_{\mathbb{R}} U\left(t-t^{\prime}\right) f\left(t^{\prime}\right) \operatorname{sign}\left(t-t^{\prime}\right) \mathrm{d} t^{\prime}+\frac{1}{2} \nabla^{2} \int_{\mathbb{R}} U\left(t-t^{\prime}\right) f\left(t^{\prime}\right) \operatorname{sign}\left(t^{\prime}\right) \mathrm{d} t^{\prime} .
$$

Denote the first term by $\nabla^{2} F(t)$. Taking a space-time Fourier transform shows that $\mathcal{F}_{t, x, y} F(\xi, \eta, \tau)=\widehat{\operatorname{sign}}\left(\tau-\xi\left(\xi^{2}+|\eta|^{2}\right)\right) \mathcal{F}_{t, x, y}(f)(\tau, \xi, \eta)$. By Plancherel, we have

$$
\left\|\nabla^{2} F\right\|_{L_{y, t}^{2}}=\left\|K(\tau, x,|\eta|) * \mathcal{F}_{y, t}(f(x, \cdot))(\eta, \tau)\right\|_{L_{\eta, \tau}^{2}},
$$

where $K(\tau, x,|\eta|)=\int_{\mathbb{R}} e^{i x \xi} \frac{\xi^{2}+|\eta|^{2}}{\tau-\xi\left(\xi^{2}+|\eta|^{2}\right)} \mathrm{d} \xi$. Notice that one of the consequences of the proof of Proposition 3.6 in [27] is that $\|K\|_{L^{\infty}\left(\mathbb{R}^{d+1}\right)}$ is uniformly bounded on $\tau, x$ and $\eta$. Therefore, an application of Young's convolution inequality together with another of Plancherel implies that

$$
\left\|\nabla^{2} F\right\|_{L_{y, t}^{2}} \lesssim\|f\|_{L_{x}^{1} L_{y, t}^{2}}
$$

This proves the asserted bound for the first term. For the second one, we simply use a combination of Proposition 3.3 with its dual version to $\tilde{f}(z, t)=f(z, t) \operatorname{sign}(t)$. An easy computation then shows that this finishes the proof.

Proposition 3.7. Let $f \in \mathcal{S}\left(\mathbb{R}^{d+1}\right)$. Then it holds that

$$
\left\|\int_{0}^{t} U\left(t-t^{\prime}\right) \Delta_{k} f\left(t^{\prime}\right) \mathrm{d} t^{\prime}\right\|_{L_{x}^{4} L_{y, T}^{\infty}} \lesssim 2^{\left(s_{d}-1\right)^{+} k}\left\|\Delta_{k} f\right\|_{L_{x}^{1} L_{y, T}^{2}},
$$

for all $k \geq 0$, where we let $s_{d}=\frac{d}{2}-\frac{1}{4}$.

Proof. Notice that, from Proposition 3.4 and the dual version of 3.3 , we get that

$$
\left\|\int_{0}^{T} U\left(t-t^{\prime}\right) \Delta_{k} f\left(t^{\prime}\right) \mathrm{d} t^{\prime}\right\|_{L_{x}^{4} L_{y, T}^{\infty}} \lesssim 2^{\left(s_{d}-1\right)^{+} k}\left\|\Delta_{k} f\right\|_{L_{x}^{1} L_{y, T}^{2}} .
$$

We now use an anitropic version of the Christ-Kiselev lemma [3], which can be found in Theorem B.3, part (i), from [1]. Indeed, our exponents meet well the conditions, since $4=\min (4, \infty)>2=\max \left(2,1, \frac{2 \cdot 1}{2}\right)$. This implies the desired retarded estimate, as wished.

3.3. Proof of the well-posedness result. Taking the previously stated linear estimates as a starting point, we create a set of norms designed to take into consideration the smoothing and maximal estimates of (1.3).

In fact, like in $\$ 2.3$, we define a set of auxiliary norms related to the estimates we have. Let then

$$
\|u\|_{\mathcal{Y}_{T}^{s}(d)}=\|u\|_{L_{T}^{\infty} H_{x, y}^{s}}+\left\|\langle\nabla\rangle^{s-s_{d}^{+}} u\right\|_{L_{x}^{4} L_{y, T}^{\infty}}+\left\|\langle\nabla\rangle^{s+1} u\right\|_{L_{x}^{\infty} L_{y, T}^{2}} .
$$

We then define the norms

$$
\|u\|_{\mathcal{X}_{T}^{s}(d)}=\left\|2^{s j}\right\| \Delta_{j} u\left\|_{\mathcal{Y}_{T}^{s}}\right\|_{\ell^{2}(\mathbb{N})},
$$


which defines the space in which we shall perform the iteration argument. Indeed, fix $k \geq 4$ and consider the Duhamel operator associated to (1.7):

$$
\Gamma_{u_{0}}(u)(t)=U(t) u_{0}+\int_{0}^{t} U\left(t-t^{\prime}\right) \partial_{x}\left(u^{k+1}\right)\left(t^{\prime}\right) \mathrm{d} t^{\prime} .
$$

We seek to prove that $\Gamma_{u_{0}}$ preserves some metric space

$$
\mathcal{E}_{a ; d}(T)=\left\{v \in \mathcal{X}_{T}^{s}(d):\|v\|_{\mathcal{X}_{T}^{s}(d)} \leq a\right\},
$$

and is, in fact, a contraction there. From Propositions 3.3 and 3.4, we have that

$$
\left\|U(t) u_{0}\right\|_{\mathcal{X}_{T}^{s}(d)} \lesssim\left\|u_{0}\right\|_{s}, \forall s \in \mathbb{R} .
$$

Thus, we are left with bounding the integral term. On the other hand, using Propositions 3.5, 3.6 and 3.7 together with the definitions of the $\mathcal{Y}_{T}^{s}(d)$ and $\mathcal{X}_{T}^{s}(d)$ norms, we see that

$$
\left\|\int_{0}^{t} U\left(t-t^{\prime}\right) \partial_{x}\left(u^{k+1}\right)\left(t^{\prime}\right) \mathrm{d} t^{\prime}\right\|_{\mathcal{X}_{T}^{s}(d)} \lesssim\left\|2^{s j}\right\| \Delta_{j}\left(u^{k+1}\right)\left\|_{L_{x}^{1} L_{y, T}^{2}}\right\|_{\ell^{2}(\mathbb{N})} .
$$

Our task is then to bound the right-hand side of (3.4). Now we follow the approach by Ribaud and Vento [26] in the two-dimensional setting.

We first notice that interpolating the second and third terms in the definition of $\|\cdot\|_{\mathcal{Y}_{T}^{s}(d)}$ gives

$$
2^{\alpha j}\left\|\Delta_{j} u\right\|_{L_{x}^{p} L_{y, T}^{q}} \lesssim\left\|\Delta_{j} u\right\|_{\mathcal{X}_{T}^{s}(d)}
$$

where $p=\frac{4}{1-\theta}, q=\frac{2}{\theta}$ and $\alpha=\left(s+\left(1+s_{d}\right) \theta-s_{d}\right)^{-}$. In particular, setting $\theta=\frac{s_{d}}{s_{d}+1}$, we have

$$
2^{s j}\left\|\Delta_{j} u\right\|_{L_{x}^{p_{1}^{+}} L_{y, T}^{q_{1}^{-}}} \lesssim\left\|\Delta_{j} u\right\|_{\mathcal{X}_{T}^{s}(d)},
$$

where $p_{1}=4\left(s_{d}+1\right), q_{1}=\frac{2\left(s_{d}+1\right)}{s_{d}}$. From this point, in analogy to 2.3 , a paraproduct decomposition of $\Delta_{j}\left(u^{k+1}\right)$ and Hölder's inequality shows that

$$
\left\|\Delta_{j}\left(u^{k+1}\right)\right\|_{L_{x}^{1} L_{y, T}^{2}} \lesssim \sum_{l \geq j-1}\left\|\Delta_{l} u\right\|_{L_{x}^{p_{1}^{+}} L_{y, T}^{q_{1}^{-}}}\left\|P_{l} u\right\|_{L_{x}^{\left(k p_{1}^{\prime}\right)^{-}} L_{y, T}^{\left(k \tilde{q}_{1}\right)^{+}}}^{k}
$$

Here we use the notation $p_{1}^{\prime}$ and $\tilde{q_{1}}$ for the positive reals so that $\frac{1}{p_{1}}+\frac{1}{p_{1}^{\prime}}=1$ and $\frac{1}{q_{1}}+\frac{1}{\tilde{q_{1}}}=\frac{1}{2}$. The first term in the product of the right-hand side of the equation above admits adequate bounds by the previous considerations, so it remains to estimate the second one.

Indeed, let $q=\frac{2}{\theta}=k \tilde{q_{1}}=2 k\left(s_{d}+1\right) \Longleftrightarrow \theta=\frac{1}{k\left(s_{d}+1\right)}$ in (3.5). This yields

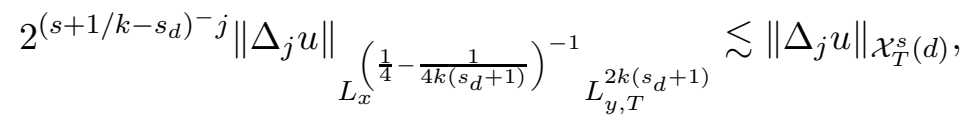

for all $j \geq 0$. Notice now that

$$
\begin{gathered}
\left\|P_{l} u\right\|_{L_{x}^{\left(k p_{1}^{\prime}\right)-} L_{y, T}^{\left(k \tilde{q_{1}}\right)+}} \lesssim \sum_{r=0}^{l}\left\|\Delta_{r} u\right\|_{L_{x}^{\left(k p_{1}^{\prime}\right)^{-}} L_{y, T}^{\left(k \tilde{q_{1}}\right)^{+}}} \\
\lesssim \sum_{r=0}^{l} 2^{(1 / 4-1 / k)^{+} r}\left\|\Delta_{r} u\right\|_{L_{x}}\left(\left(\frac{\left.\left.1-\frac{1}{4}-\frac{1}{4 k\left(s_{d}+1\right)}\right)^{-1}\right)^{+}}{L_{y, T}^{2 k\left(s_{d}+1\right)^{+}}}\right.\right.
\end{gathered}
$$


by Sobolev embedding on the $x$-variable. Notice that we need $k \geq 4$ in order for this step to work.

The right-hand side of (3.7) above is bounded by $T^{\delta}\|u\|_{\mathcal{X}_{T}^{s}(d)}$ as long as $s+\frac{1}{k}-s_{d}>$ $\frac{1}{4}-\frac{1}{k} \Longleftrightarrow s>\frac{d}{2}-\frac{2}{k}$. Indeed, this follows from interpolating (3.6) with the trivial bound

$$
2^{-j^{+}}\left\|\Delta_{j} u\right\|_{L_{x, y, T}^{N}} \lesssim T^{\delta}\left\|\Delta_{j} u\right\|_{L_{T}^{\infty} L_{x, y}^{2}}
$$

where we take $N \gg 1$ sufficiently large. Collecting these bounds, it holds that

$$
\left\|2^{s j}\right\| \Delta_{j}\left(u^{k+1}\right)\left\|_{L_{x}^{1} L_{y, T}^{2}}\right\|_{\ell^{2}(\mathbb{N})} \lesssim\left\|\left(1_{j \geq 0} 2^{-s j}\right) *_{j}\right\| \Delta_{j} u\left\|_{\mathcal{X}_{T}^{s}(d)}\right\|_{\ell^{2}(\mathbb{N})}\|u\|_{\mathcal{X}_{T}^{s}(d)}^{k} .
$$

By the discrete version of Young's convolution inequality, the latter term is controlled by $\|u\|_{\mathcal{X}_{T}^{s}(d)}$. Therefore,

$$
\left\|\Gamma_{u_{0}}(u)\right\|_{\mathcal{X}_{T}^{s}(d)} \leq C_{s}\left\|u_{0}\right\|_{H^{s}}+C \cdot T^{\delta}\|u\|_{\mathcal{X}_{T}^{s}(d)}^{k+1},
$$

and thus, for $a=2 C_{s}\left\|u_{0}\right\|_{H^{s}}$ and $T \sim_{s}\left(1+\left\|u_{0}\right\|_{H^{s}}\right)^{-\beta_{s}}$, for some $\beta_{s}>0$, it holds that $\Gamma_{u_{0}}$ maps $\mathcal{E}_{a ; d}(T)$ to itself. Moreover, redoing all the computations above with $\Gamma_{u_{0}} u-\Gamma_{u_{0}} v$ yields that it is, in fact, a contraction on such space for such $T$. Therefore, it has a unique fixed point, which is our desired solution.

By the standard-by-now methods, we conclude that this solution is unique and, by the fact that $\Gamma_{u_{0}}$ was Lipschitz, we conclude that the data-to-solution map is, in fact, locally Lipschitz on $C\left([0, T]: H^{s}\right) \cap \mathcal{X}_{T}^{s}(d)$, as desired.

3.4. Pointwise convergence of the flow. In this subsection, we discuss the proof of Theorem 1.5. Indeed, we begin with an approximation lemma, which in turn is based off [5. Proposition 3.3]. In what follows, we denote by $u_{N}$ the (unique) solution to

$$
\begin{cases}\partial_{t} u_{N}+\partial_{x} \Delta u_{N}+\partial_{x}\left(P_{N}\left(u_{N}^{k+1}\right)\right)=0 & \text { on } \mathbb{R}^{d} \times \mathbb{R} \\ u_{N}(x, 0)=P_{N} u_{0}(x), & \text { on } \mathbb{R}^{d} .\end{cases}
$$

By the energy method, for instance, we can see that solutions to (3.8) are smooth, as the initial data $P_{N} u_{0}$ is smooth for any $u_{0} \in H^{s}$. This fact will be crucial in the proofs below.

We start by proving that the $L^{4}$-maximal-in-time estimate for the group $U(t)$ fulfills our purposes whenever $d=2$ and $k \geq 2, d=3, k \geq 3$ or $d \geq 3, k \geq 4$.

Lemma 3.8. Suppose that, for $u_{0} \in H^{s}\left(\mathbb{R}^{d}\right)$, we have that

$$
\left\|u_{N}-u\right\|_{L_{x, y}^{4} L_{T}^{\infty}} \rightarrow 0 \text { as } N \rightarrow \infty .
$$

Then it holds that $u(z, t) \rightarrow u_{0}(z)$ as $t \rightarrow 0$ for almost every $z \in \mathbb{R}^{d}$.

Proof. The proof is another instance of the relationship between maximal functions and pointwise convergence. Indeed, by smoothness of $u_{N}$, it holds that

$$
u_{N}(z, t) \rightarrow P_{N} u_{0}(z) \text { for all } z \in \mathbb{R}^{d} .
$$

Therefore,

$$
\limsup _{t \rightarrow 0}\left|u(z, t)-u_{0}(z)\right| \leq \limsup _{t \rightarrow 0}\left|u(z, t)-u_{N}(z, t)\right|+\left|\left(I-P_{N}\right) u_{0}(z)\right| .
$$


Thus, by Chebyshev's inequality,

$$
m\left(\left\{z \in \mathbb{R}^{d}: \limsup _{t \rightarrow 0}\left|u(z, t)-u_{0}(z)\right|>\varepsilon\right\}\right) \leq \frac{1}{\varepsilon^{4}}\left\|u-u_{N}\right\|_{L_{x, y}^{4} L_{T}^{\infty}}^{4}+\frac{1}{\varepsilon^{2}}\left\|\left(I-P_{N}\right) u_{0}\right\|_{L^{2}}^{2} .
$$

Notice that the two terms in the right-hand side above can be made arbitrarily small by letting $N \rightarrow \infty$. Thus, we conclude that $m\left(\left\{z \in \mathbb{R}^{d}: \lim _{\sup _{t \rightarrow 0}}\left|u(z, t)-u_{0}(z)\right|>\varepsilon\right\}\right)=$ $0, \forall \varepsilon>0$. This concludes the proof.

Proof of Theorem 1.5, $d \geq 2, k \geq d$ case. We start by writing the Duhamel formulation of both (1.7) and (3.8). It gives us that

$$
\left\|u_{N}-u\right\|_{L_{x, y}^{4} L_{T}^{\infty}} \lesssim\left\|u_{0}-P_{N} u_{0}\right\|_{H^{s_{d}^{+}}}+\left\|\int_{0}^{t} U\left(t-t^{\prime}\right) \partial_{x}\left(P_{N}\left(u_{N}^{k+1}\right)-u^{k+1}\right)\left(t^{\prime}\right) \mathrm{d} t^{\prime}\right\|_{L_{x, y}^{4} L_{T}^{\infty}}
$$

where we used Proposition (3.4) in the first term. In order to control the integral term, we observe that in the same way we proved Proposition 3.7, it holds that,

$$
\left\|\int_{0}^{t} U\left(t-t^{\prime}\right) \partial_{x} \Delta_{j} f\left(t^{\prime}\right) \mathrm{d} t^{\prime}\right\|_{L_{x, y}^{4} L_{T}^{\infty}} \lesssim 2^{\tilde{s}_{d}^{+} j}\left\|\Delta_{j} f\right\|_{L_{x}^{1} L_{y, T}^{2}} .
$$

Thus, for all $s>\tilde{s}_{d}$, the integral term $\left\|\int_{0}^{t} U\left(t-t^{\prime}\right) \partial_{x}\left(P_{N}\left(u_{N}^{k+1}\right)-u^{k+1}\right)\left(t^{\prime}\right) \mathrm{d} t^{\prime}\right\|_{L_{x, y}^{4} L_{T}^{\infty}}$ is controlled by

$$
\begin{aligned}
C_{s}\left\|2^{s j}\right\| \Delta_{j}\left(P_{N}\left(u_{N}^{k+1}\right)-u^{k+1}\right)\left\|_{L_{x}^{1} L_{y, T}^{2}}\right\|_{\ell_{j}^{2}} \lesssim & \left\|2^{s j}\right\| \Delta_{j}\left(P_{N}\left(u_{N}^{k+1}-u^{k+1}\right)\right)\left\|_{L_{x}^{1} L_{y, T}^{2}}\right\|_{\ell_{j}^{2}} \\
& +\left\|2^{s j}\right\| \Delta_{j}\left(\left(I-P_{N}\right) u^{k+1}\left\|_{L_{x}^{1} L_{y, T}^{2}}\right\|_{\ell_{j}^{2}} .\right.
\end{aligned}
$$

Now the first term on the right-hand side of (3.10) is controlled, by Young's inequality, by $\left\|u-u_{N}\right\|_{\mathcal{X}_{T}^{s}(d)}$, with the definition of the $\mathcal{X}_{T}^{s}(d)$ spaces we have adopted throughout the text, as $s>\max \left(\frac{d}{2}-\frac{2}{k}, \tilde{s}_{d}\right)$. On the other hand, it is easy to see from the monotone convergence theorem, together with the proofs of Theorem 1.4, Theorem 4.2 and Theorem 1.1 in [26], that the second term on the right-hand side of (3.10) goes to 0 as $N \rightarrow \infty$. Inserting back into (3.9), we obtain, for $N \gg 1$,

$$
\left\|u_{N}-u\right\|_{L_{x, y}^{4} L_{T}^{\infty}} \lesssim_{s} \delta+\left\|u_{N}-u\right\|_{\mathcal{X}_{T}^{s}(d)} .
$$

On the other hand, by Theorem 1.4, for $N$ sufficiently large and $T=T\left(\left\|u_{0}\right\|_{s}\right)$, it holds that

$$
\left\|u_{N}-u\right\|_{\mathcal{X}_{T}^{s}(d)} \lesssim_{s}\left\|\left(I-P_{N}\right) u_{0}\right\|_{H^{s}} \lesssim_{s} \delta .
$$

This promptly implies that $\left\|u_{N}-u\right\|_{L_{x, y}^{4} L_{T}^{\infty}}$ can be made arbitrarily small as $N \rightarrow \infty$, given $s>\max \left(\frac{d}{2}-\frac{2}{k}, \tilde{s}_{d}\right)$. The conditions of Lemma 3.8 are then met, and we have concluded the proof.

In order to handle the $d=3, k=2$ case, we remark that another maximal estimate with bounds independent of the dimension holds in the case of the group $U(t)$. In fact, a result by Cowling [4] has as by-product that, whenever $\Omega: \mathbb{R}^{d} \rightarrow \mathbb{R}$ is smooth and homogeneous 
of degree $m$, then

$$
\left\|\sup _{t \in[0,1]}\left|e^{i t \Omega(D)} f\right|\right\|_{L^{2}\left(\mathbb{R}^{d}\right)} \lesssim\|f\|_{H^{s}},
$$

whenever $s>m / 2$. In our case, we obtain that the global bound

$$
\|U(t) f\|_{L^{2}\left(\mathbb{R}^{d}\right) L_{[0,1]}^{\infty}} \lesssim\|f\|_{H^{s}}
$$

holds whenever $s>\frac{3}{2}$. By Roger's local-to-global transference principle (see Theorem B), we have that the local maximal bound

$$
\|U(t) f\|_{L^{2}\left(B^{d}(0,1)\right) L_{[0,1]}^{\infty}} \lesssim\|f\|_{H^{s}}
$$

holds for all $s>\frac{1}{2}$ and all dimensions $d \geq 2$. We will use this local maximal bound in the three-dimensional case of the modified Zakharov-Kuznetsov equation.

Lemma 3.9. Let $u, u_{N}$ denote solutions to (1.7) and (3.8), respectively, in the case $k=$ $2, d=3$. Suppose that, for $u_{0} \in H^{s}\left(\mathbb{R}^{3}\right)$, we have the existence of $T=T\left(\left\|u_{0}\right\|_{s}\right)$ so that

$$
\left\|u_{N}-u\right\|_{L^{2}\left(B^{3}(0,1)\right) L_{T}^{\infty}} \rightarrow 0 \text { as } N \rightarrow \infty .
$$

Then it holds that $u(z, t) \rightarrow u_{0}(z)$ as $t \rightarrow 0$ for almost every $z \in \mathbb{R}^{3}$.

Proof. The proof of this lemma is almost identical to that of Lemma 3.8, only that this time we employ the Chebyshev inequality argument on a fixed unit ball of $\mathbb{R}^{3}$, use the translation invariance of the equations involved and cover the euclidean space $\mathbb{R}^{3}$ by countably many such balls. We omit the details.

Proof of Theorem 1.5, $d=3, k=2$ case. By [31, Lemma 2.9] (see also [5, Lemma 2.1] and the comments thereafter), the maximal estimate (3.11) implies the continuous embedding $X_{\delta}^{s, b} \hookrightarrow L^{2}\left(B^{3}(0,1)\right) L_{[0, \delta]}^{\infty}$ for the Bourgain space $X_{\delta}^{s, b}$ when $s, b>\frac{1}{2}$, where we define the norm

$$
\|F\|_{X^{s, b}}=\left\|\langle(\xi, \eta)\rangle^{s}\left\langle\tau-\xi\left(\xi^{2}+|\eta|^{2}\right)\right\rangle^{b} \widehat{u}(\xi, \eta, \tau)\right\|_{L_{\xi, \eta, \tau}^{2}},
$$

where we have used the space-time Fourier transform above. Thus, it holds that

$$
\left\|\sup _{t \in[0, \delta]}|F(x, t)|\right\|_{L^{2}\left(B^{3}(0,1)\right)} \lesssim\|F\|_{X_{\delta}^{s, b}}, \forall F \in X_{\delta}^{s, b} .
$$

This readily implies that

$$
\left\|u-u_{N}\right\|_{L_{B^{3}}^{2} L_{T}^{\infty}} \lesssim\left\|u-u_{N}\right\|_{X_{T}^{s, b}}
$$

By the Duhamel principle applied to $u, u_{N}$ and the properties of the Bourgain spaces $X_{T}^{s, b}$, we see that

$$
\begin{aligned}
& \left\|u-u_{N}\right\|_{X_{T}^{s, b}} \\
& \left.\quad \lesssim\left\|\left(I-P_{N}\right) u_{0}\right\|_{H^{s}\left(\mathbb{R}^{3}\right)}+\| P_{N} \partial_{x}\left(u^{3}-\left(u_{N}\right)^{3}\right)\right)\left\|_{X_{T}^{s, b^{\prime}}}+\right\|\left(I-P_{N}\right) \partial_{x}\left(u^{3}\right) \|_{X_{T}^{s, b^{\prime}}},
\end{aligned}
$$

for some $b^{\prime}>-\frac{1}{2}$. Grünrock's trilinear estimate [12, Proposition 1] for the modified Zakharov-Kuznetsov equation in dimension three implies then that, for each $s>\frac{1}{2}$, there 
must be $b^{\prime}>-\frac{1}{2}$ so that for all $b>\frac{1}{2}$,

$$
\left\|\partial_{x}\left(u^{3}-v^{3}\right)\right\|_{X_{T}^{s, b^{\prime}}} \lesssim T^{\delta}\left(\|u\|_{X_{T}^{s, b}}^{2}+\|v\|_{X_{T}^{s, b}}^{2}\right)\|u-v\|_{X_{T}^{s, b}}
$$

The first consequence of (3.13) is by setting $v=u_{N}$, which shows that

$$
\left.\| P_{N} \partial_{x}\left(u^{3}-\left(u_{N}\right)^{3}\right)\right)\left\|_{X_{T}^{s, b^{\prime}}} \lesssim T^{\delta} C\left(\left\|u_{0}\right\|_{s}\right)\right\| u-u_{N} \|_{X_{T}^{s, b}}
$$

By taking $T$ sufficiently small, it holds that the right-hand side of the expression above can be absorbed into the left-hand side of (3.12). As a second consequence, setting $v \equiv 0$ on (3.13) and using the definition of $X_{T}^{s, b}$, we have that

$$
\left\|\left(I-P_{N}\right) \partial_{x}\left(u^{3}\right)\right\|_{X_{T}^{s, b}} \rightarrow 0 \text { as } N \rightarrow \infty .
$$

Therefore, as $u_{0} \in H^{s}, s>\frac{1}{2}$, we see that the left-hand side of (3.12) converges to 0 as $N \rightarrow \infty$. This finishes this case by Lemma 3.9, and thus also the proof of Theorem [1.5.

\section{Comments and Remarks}

4.1. Sharp maximal estimates in two dimensions. As discussed in $\sqrt{2}$, the spacetime maximal estimates (1.4) are, in fact, sharp for the three-dimensional case, due to our counterexamples and the previous works [27, 12].

On the other hand, although we have made progress in the question of sharpness of space-time maximal estimates in dimension 2 through simple counterexamples as well as through the indirect method given in (2.3), we still have a gap where next to nothing is known about sharpness. Indeed, for $p \in(2,4)$, we only know that the estimate

$$
\left\|U(t) u_{0}\right\|_{L_{x}^{p} L_{y, T}^{\infty}} \lesssim\left\|u_{0}\right\|_{s}
$$

holds, by interpolation, in the $s>\frac{3}{4}$ range. It is likely, however, that this is not the sharp range for those indices; in fact, we conjecture the following:

Conjecture 4.1. If $d=2, p=3$, then (4.1) holds for all $s>\frac{2}{3}$.

A hand-waving justification for such a conjecture is the following: by the results in [13, local well-posedness for the quartic Zakharov-Kuznetsov equation in two dimensions holds in $H^{s}$, whenever $s>\frac{1}{3}$. On the other hand, considering the modified norms

$$
\|u\|_{\tilde{Y}_{T}^{s}}=\|u\|_{L_{T}^{\infty} L_{x, y}^{2}}+\left\|\langle\nabla\rangle^{s-\frac{2}{3}^{+}} u\right\|_{L_{x}^{3} L_{y, T}^{\infty}}+\left\|\langle\nabla\rangle^{s+1} u\right\|_{L_{x}^{\infty} L_{y, T}^{2}},
$$

with $\|u\|_{\tilde{X}_{T}^{s}}=\left\|2^{s j}\right\| \Delta_{j} u\left\|_{\tilde{Y}_{T}^{s}}\right\|_{\ell^{2}(\mathbb{N})}$, and reproducing the argument in 92.3 , we see that if (4.1) holds for all $s>\frac{2}{3}$, we recover the full range $s>\frac{1}{3}$ of local well-posedness.

For other values of $p \in(2,4)$, it is not crystal clear what should happen. In fact, when $p \in(3,4)$, Conjecture 4.1 plus Proposition 1.1 would imply that those estimates are, in fact, sharp for the interpolation between the $L_{x}^{4} L_{y, T}^{\infty}$ and the $L_{x}^{3} L_{y, T}^{\infty}$. On the range $p \in(2,3)$, on the other hand, we do not know what to expect: on the one hand, the range should "blow up" to $s>\frac{3}{4}$ as $p \rightarrow 2$; on the other hand, it is not, in principle, impossible for the range to be the necessary one given by Proposition 1.1 up until the $p=2$ endpoint. 
4.2. Maximal estimates and LWP for the $k=3$ case. As previously remarked, the Strichartz estimate

$$
\left\|U(t) u_{0}\right\|_{L_{x, y, t}^{4}} \lesssim\left\|u_{0}\right\|_{H^{s}}, s>\frac{d-3}{4},
$$

only allows us to prove local well-posedness for the IVP 1.7 if $k \geq 4$, and provides us with the full subcritical range of results in such cases by passing to suitable maximal functions. Together with the $k=1$ case in [15] and $k=2$ in [19], the only remaining case for proving local well-posedness for the generalized Zakharov-Kuznetsov equation in higher dimensions is $k=3$. For $d=2,3$, such a result was obtained by Grünrock by using a suitable modification of the Ribaud-Vento techniques.

One of the main features of Grünrock's proof is the use of Strichartz estimates with derivative gain. In fact, he is able to reach the full subcritical range by using the estimates

$$
\left\|K\left(D_{x}, D_{y}\right)^{1 / 8} U(t) u_{0}\right\|_{L_{x, y, t}^{4}\left(\mathbb{R}^{3}\right)} \lesssim\left\|u_{0}\right\|_{L^{2}\left(\mathbb{R}^{2}\right)},
$$

where we define $K\left(D_{x}, D_{y}\right)^{\sigma} g=\mathcal{F}_{x, y}^{-1}\left|3 \xi^{2}-\eta^{2}\right|^{\sigma} \mathcal{F}_{x, y} g$, and

$$
\left\|D_{x}^{1 / 10} U(t) u_{0}\right\|_{L^{\frac{15}{4}\left(\mathbb{R}^{4}\right)}} \lesssim\left\|u_{0}\right\|_{L^{2}\left(\mathbb{R}^{3}\right)} .
$$

Unfortunately, such estimates seem not to be available in higher dimensions, with an exception for the Kato Smoothing estimate in Proposition 3.3. Instead of these, one may use the maximal estimates, as illustrated by our method, in order to reach the sharp bounds. Indeed, as the space-time maximal estimate (1.4) is sharp in three dimensions, the argument in Section 3 above can be refined to prove local well-posedness for the whole subcritical range $s>\frac{3}{2}-\frac{2}{k}$ in three dimensions for all $k \geq 2$.

As a matter of fact, we only need to redefine the norms defining the spaces $\mathcal{X}_{T}^{s}(3)$ as follows: let first

$$
\|u\|_{\tilde{\mathcal{Y}}_{T}^{s}(3)}=\|u\|_{L_{T}^{\infty} L_{y, T}^{2}}+\left\|\left\langle\nabla_{x, y}\right\rangle^{s-1^{+}} u\right\|_{L_{x}^{2} L_{y, T}^{\infty}}+\left\|\left\langle\nabla_{x, y}\right\rangle^{s+1} u\right\|_{L_{x}^{\infty} L_{y, T}^{2}},
$$

and then $\|u\|_{\tilde{\mathcal{X}}_{T}^{s}(3)}=\left\|2^{s j}\right\| \Delta_{j} u\left\|_{\tilde{\mathcal{Y}}_{T}^{s}(3)}\right\|_{\ell^{2}(\mathbb{N})}$. The computations performed in the proof of Theorem 1.4 adapt easily due to the sharp bound

$$
\left\|U(t) u_{0}\right\|_{L_{x}^{2} L_{y, T}^{\infty}} \lesssim\left\|u_{0}\right\|_{s}, s>1
$$

and we reobtain the following result:

Theorem 4.2 (Main result in [12]; Three-dimensional result in [13]). Let $d=3$ and $k \in\{2,3\}$. Then there are function spaces $\tilde{\mathcal{X}}_{T}^{s}$ so that for each $u_{0} \in H^{s}\left(\mathbb{R}^{3}\right)$ with $s>\frac{3}{2}-\frac{2}{k}$, the IVP (1.7) has a unique solution

$$
u \in C\left([0, T]: H^{s}\right) \cap \tilde{\mathcal{X}}_{T}^{s},
$$

where $T=T\left(\left\|u_{0}\right\|_{s}\right)>0$. Moreover, the map $u_{0} \mapsto u$ from $H^{s}\left(\mathbb{R}^{3}\right)$ to $\tilde{\mathcal{X}}_{T}^{s} \cap C\left([0, T]: H^{s}\right)$ is locally Lipschitz continuous.

As mentioned previously, we (still) do not know whether the conjectured sharp bound

$$
\left\|U(t) u_{0}\right\|_{L_{x}^{2} L_{y, T}^{\infty}} \lesssim\left\|u_{0}\right\|_{s}, s>\frac{d-1}{2}
$$


holds in order to adapt the idea given above to the high-dimensional setting. Nevertheless, the Sobolev embedding theorem easily implies that

$$
\left\|U(t) u_{0}\right\|_{L_{x}^{2} L_{y, T}^{\infty}} \lesssim\left\|\langle\nabla\rangle^{\frac{d-1}{2}} U(t) u_{0}\right\|_{L_{x, y}^{2} L_{T}^{\infty}} \lesssim\left\|\left(\partial_{x} \Delta\right)^{\frac{1^{+}}{2}}\langle\nabla\rangle^{\frac{d-1}{2}^{+}} U(t) u_{0}\right\|_{L_{x, y, T}^{2}},
$$

which, on the other hand, is bounded by $T^{1 / 2}\left\|u_{0}\right\|_{H^{s}}$, whenever $s>\frac{d+2}{2}$. By defining the norm

$$
\|u\|_{\tilde{\mathcal{Y}}_{T}^{s}(d)}=\|u\|_{L_{T}^{\infty} L_{y, T}^{2}}+\left\|\left\langle\nabla_{x, y}\right\rangle^{s-\left(\frac{d+2}{2}\right)^{+}} u\right\|_{L_{x}^{2} L_{y, T}^{\infty}}+\left\|\left\langle\nabla_{x, y}\right\rangle^{s+1} u\right\|_{L_{x}^{\infty} L_{y, T}^{2}}
$$

and consequently $\|u\|_{\tilde{\mathcal{X}}_{T}^{s}(d)}=\left\|2^{s j}\right\| \Delta_{j} u\left\|_{\mathcal{Y}_{T}^{(\tilde{s})}(d)}\right\|_{\ell^{2}(\mathbb{N})}$, the aforementioned techniques to set the Picard iteration scheme in motion imply immediately the following result:

Theorem 4.3. For $d \geq 4, k=3$, we have that for each $u_{0} \in H^{s}\left(\mathbb{R}^{d}\right)$ with $s>\frac{d}{2}+\frac{5}{6}$, the IVP (1.7) has a unique solution

$$
u \in C\left([0, T]: H^{s}\right) \cap \tilde{\mathcal{X}}_{T}^{s},
$$

where $T=T\left(\left\|u_{0}\right\|_{s}\right)>0$. Moreover, the map $u_{0} \mapsto u$ from $H^{s}\left(\mathbb{R}^{3}\right)$ to $\tilde{\mathcal{X}}_{T}^{s} \cap C\left([0, T]: H^{s}\right)$ is locally Lipschitz continuous.

Currently, we believe that the estimate (4.2) holds for all $d \geq 3$. Nonetheless, we also believe that the use of (4.2) is not strictly necessary in order to prove local well-posedness in the full subcritical range $s>\frac{d}{2}-\frac{2}{k}$. Indeed, Kinoshita's method for proving local well-posedness (and, in fact, also global well-posedness on the critical Sobolev space for small data) only uses a bilinear estimate and Strichartz estimates for the group $U(t)$; therefore, it might be possible to reach the full range by employing Proposition 1.3 and other non-endpoint inequalities.

\section{ACKNOWLEDGEMENTS}

F. L. was partially supported by CNPq and FAPERJ, Brazil. J.P.G.R. acknowledges financial support from CNPq, Brazil.

\section{REFERENCES}

[1] W. Baoxiang, H. Lijia and H. Chunyan, Global well-posedness and scattering for the derivative nonlinear Schrödinger equation with small rough data. Ann. Inst. H. Poincaré - AN. 26 (2009), 2253-2281.

[2] H. Biagioni and F. Linares, Well-posedness results for the modified Zakharov-Kuznetsov equation. in Nonlinear Equations: Methods, Models and Applications, Progr. Nonlinear Differential Equations Appl., 54 (2003), Birkhäuser, Basel, 181-189.

[3] M. Christ and A. Kiselev, Maximal functions associated to filtrations. J. Func. Anal. 179 (2001), n. $2,409-425$.

[4] M. Cowling, Pointwise behavior of solutions to Schrödinger equations. in:Harmonic Analysis, Cortona, 1982. Lecture Notes in Math., 992, Springer, Berlin, 1983, 83-90.

[5] E. Compaan, R. Lucà and G. Staffilani, Pointwise Convergence of the Schrödinger flow. Int. Math. Res. Notices (in press).

[6] X. Du, L. Guth and X. Li, A sharp Schrödinger maximal estimate in $\mathbb{R}^{2}$. Ann. of Math. 186 (2017), n. $2,607-640$.

[7] X. Du and R. Zhang, Sharp $L^{2}$ estimates of the Schrödinger maximal function in higher dimensions. Ann. of Math. 189 (2019), n. 3, 837-861. 
[8] M.B. Erdoğan and N. Tzirakis, Global smoothing for the periodic KdV evolution. Int. Math. Res. Notices 20 (2013), 4589-4614.

[9] M.B. Erdoğan and N. Tzirakis, Talbot effect for the cubic non-linear Schrödinger equation on the torus. Math. Res. Lett. 20 (2013), n. 6 , 1081-1090.

[10] A. V. Faminskii, The Cauchy problem for the Zakharov-Kuznetsov equation. Differentsial' nye Uravneniya 31 (1995), n. 6, 1070-1081.

[11] L.G. Farah, F. Linares and A. Pastor, A note on the 2D generalized Zakharov-Kuznetsov equation: Local, global, and scattering results. J. Diff. Eqs. 253 (2012), n. 8, 2558-2571.

[12] A. Grünrock, A Remark on the modified Zakharov-Kuznetsov equation in three space dimensions. Math. Res. Lett. 21 (2014), n. 1, 127-131.

[13] A. Grünrock, On the generalized Zakharov-Kuznetsov equation at critical regularity, preprint, arXiv:1509.09146.

[14] A. Grünrock and S. Herr, The Fourier restriction norm method for the Zakharov-Kuznetsov equation. Disc. Cont. Din. Syst. 34 (2014), n. 5, 2061-2068.

[15] S. Herr and S. Kinoshita, Subcritical well-posedness results for the Zakharov-Kuznetsov equation in dimension three and higher. arXiv preprint: arXiv:2001.09047.

[16] J. Hickman, F. Linares, O. Riaño, K. Rogers and J. Wright, On a higher dimensional version of the Benjamin-Ono equation. SIAM J. Math. Anal. 51 (2019), n. 6, 4544-4569.

[17] C. E. Kenig, G. Ponce, and L. Vega, Oscillatory integrals and regularity of dispersive equations. Indiana Univ. Math. J., 40 (1991), 33-69.

[18] S. Kinoshita, Global Well-posedness for the Cauchy problem of the Zakharov-Kuznetsov equation in 2D. arXiv preprint: arXiv:1905.01490.

[19] S. Kinoshita, Well-posedness for the Cauchy problem of the modified Zakharov-Kuznetsov equation. arXiv preprint: arXiv:1911.13265.

[20] D. Lannes, F. Linares and J.-C. Saut, The Cauchy problem for the Euler-Poisson system and derivation of the Zakharov-Kuznetsov equation. Prog. Non-linear Diff. Eqs Appl. 84 (2013), 181-213.

[21] F. Linares and A. Pastor, Local and global well-posedness for the 2D generalized Zakharov-Kuznetsov equation. J. Funct. Anal. 260 (2011), 1060-1085.

[22] F. Linares and A. Pastor, Well-posedness for the two-dimensional modified Zakharov-Kuznetsov equation. SIAM J. Math. Anal. 41 (2009), No. 4, 1323-1339.

[23] F. Linares and J.P.G. Ramos, The Cauchy problem for the $L^{2}$-critical generalized Zakharov-Kuznetsov equation in dimension 3. preprint.

[24] F. Linares and J.-C. Saut, The Cauchy problem for the 3D Zakharov-Kuznetsov equation. Disc. Cont. Dyn. Systems - A 24 (2009), n. 2, 547-565.

[25] L. Molinet and D. Pilod, Bilinear Strichartz estimates for the Zakharov-Kuznetsov equation and applications. Annales de l'Institut Henri Poincare (C) Non Linear Analysis 32 (2015), n. 2, 347-371.

[26] F. Ribaud and S. Vento, A Note on the Cauchy problem for the 2D generalized Zakharov-Kuznetsov equations. C. R. Acad. Sci. Paris, Ser. I, 350 (2012), 499-503.

[27] F. Ribaud and S. Vento, Well-posedness results for the three-dimensional Zakharov-Kuznetsov equation. SIAM J. Math. Anal. 44 (2012), n. 4, 2289-2304.

[28] K. M. Rogers, A local smoothing estimate for the Schrödinger equation. Adv. Math. 219 (2008), $2105-2122$.

[29] R. Schippa, On the Cauchy problem for higher dimensional Benjamin-Ono and Zakharov-Kuznetsov equations. arXiv preprint: arXiv:1903.02027.

[30] P. Sjölin, A Counter-example Concerning Maximal Estimates for Solutions to Equations of Schrödinger Type. Indiana Univ. Math. J. 47 (1988), n. 2, 593-599.

[31] T. Tao, Nonlinear Dispersive Equations: Local and Global Analysis. NSF-CBMF Regional Conference Series in Mathematics, Number 106.

[32] V. E. Zakharov and E. A. Kuznetsov, On three dimensional solitons. Sov. Phys. JETP. 39 (1974) $285-286$. 
(F. Linares) impa, Instituto Matemática Pura e Aplicada, Estrada Dona Castorina 110, 22460-320, Rio DE JANEIRO, RJ, BRAZIL

E-mail address: linares@impa.br

(J.P. Ramos) imPa, Instituto Matemática Pura e Aplicada, Estrada Dona Castorina 110, 22460-320, Rio de Janeiro, RJ, BraziL

E-mail address: joaopgramos95@gmail.com 